\section{Proton Transfer Mass}

\section{Spectrometer}

Armin Hansel, Associate Professor at the Institut für Ionenphysik, University of Innsbruck, Austria

$T$ he first Proton Transfer Reaction Mass Spectrometer (PTRMS) instrument was built ten years ago in our laboratories at the Institute of Ion Physics of the University of Innsbruck. In PTR-MS we use $\mathrm{H}_{3} \mathrm{O}^{+}$ions to ionise volatile organic compounds (VOC) present in gaseous media, e.g. in air. This technique enables a variety of organic species in complex matrices to be monitored in real-time, with detection limits as low as a few parts per trillion, volume (pptv). In 1998 we founded the spin-off company Ionicon Analytik GmbH to provide this technique to a growing user community. Today we manufacture and sell PTRMS instruments throughout the world. Our customers include noted multinational companies and renowned research institutions in the fields of environmental and food technology. This review covers the main principles of PTR-MS and shows key academic and commercial application areas in the market place.

\section{Why PTR-MS?}

Conventional mass spectrometry (MS) is a well proven and highly sensitive technique for the identification and detection of organic pollutants. In simple terms, it works by separating organic molecules on the basis of their molecular masses. Molecules entering the mass spectrometer are ionised, usually by electron impact, and are then subjected to electromagnetic fields under whose influence ions with different mass/charge ratios will move in different trajectories. Thus the ionised molecules can be separated and individual molecules can be identified. That is the theory. Unfortunately, instead of forming a single ionised species, many molecules break down into smaller fragments, each of which is detected separately. This can result in one compound giving rise to a complex "mass spectrum". With a mixture of compounds entering the MS detector simultaneously, the final

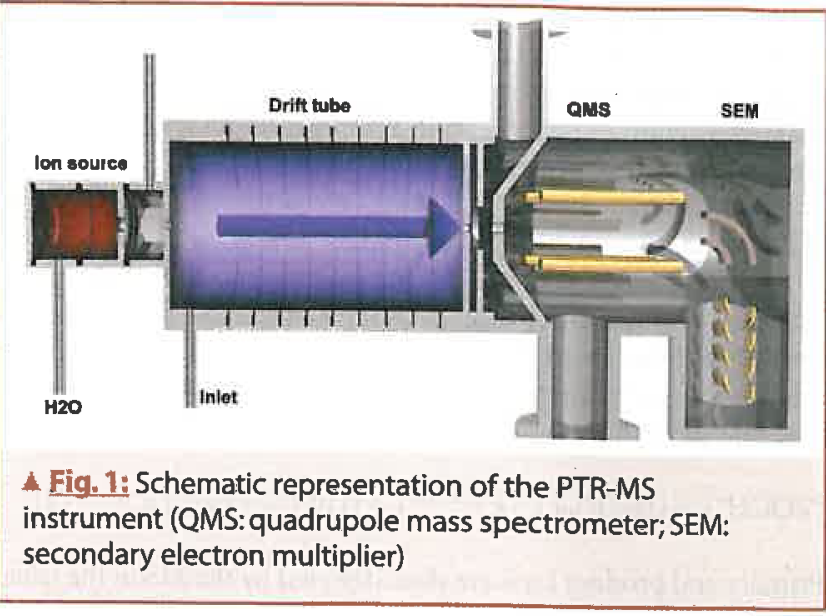

mass spectrum may be so complex that interpretation and quantification become difficult, if not impossible. The traditional solution to this problem has been to separate the compounds with a gas chromatograph (GC) before they are sent to a mass spectrometer. Unfortunately, GCs are inherently slow - a typical separation of just one sample could take 30 minutes - so while GC-MS is fine for analysing discrete samples or monitoring slowly changing situations, it cannot usually be regarded as a "real time" or on-line technique. PTR-MS can make the GC step unnecessary for many typical VOC analyses and achieve a response time measured in milliseconds. This opens a whole new range of possibilities in environmental monitoring, food science, odour analysis and medical applications.

\section{How does it work?}

The fundamental difference between a conventional MS and PTRMS is the "soft ionisation" method used to ionise the organic molecules. PTR-MS uses chemical ionisation, in which the VOC molecules react with charged ions, in this case hydroxonium ions $\left(\mathrm{H}_{3} \mathrm{O}^{+}\right)$produced in an external ion source. The first PTR-MS instrument was developed at the University of Innsbruck [1-2] and is shown schematically in Figure 1. A glow discharge acts as an external ion source which produces $\mathrm{H}_{3} \mathrm{O}^{+}$ions from pure water vapour. $\mathrm{H}_{3} \mathrm{O}^{+}$primary ions enter the drift tube (DT), which is flushed continuously with ambient air, and undergo non-reactive
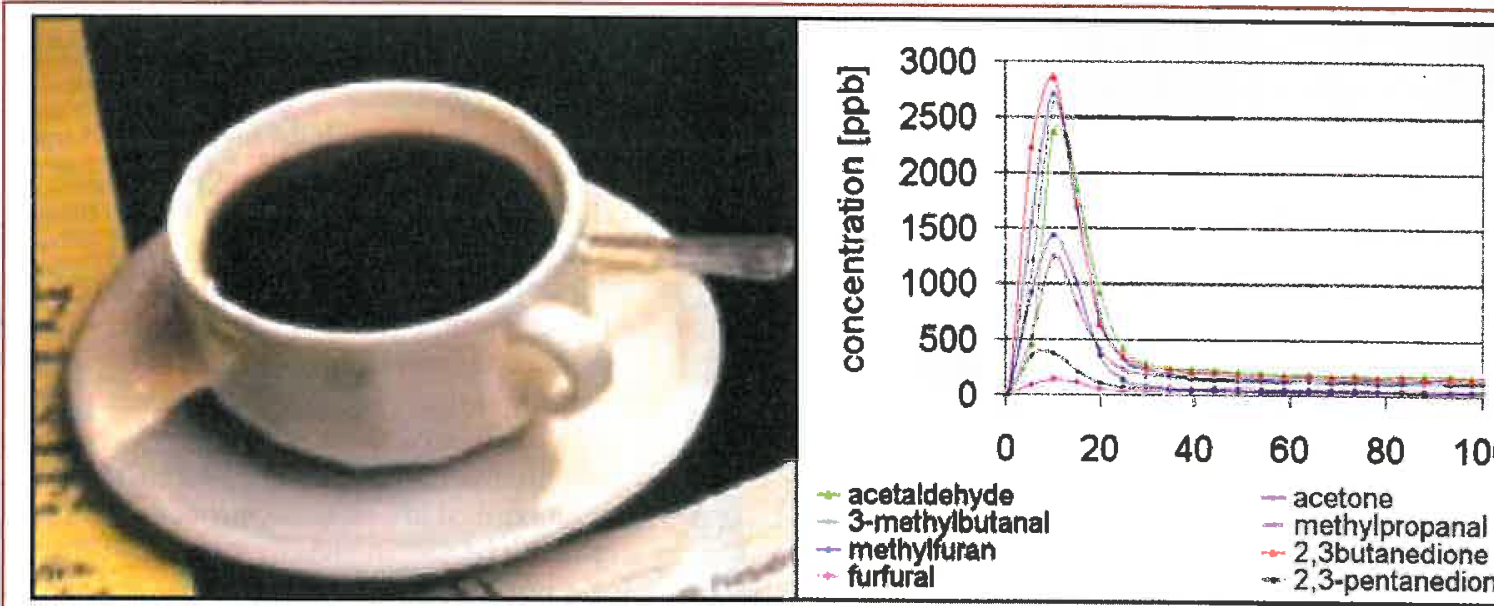

20

40

$60 \quad 80$

100 time [s]

- acetone

- methylpropanal

- 2,3butanedione 2methylbutanal

2,3-pentanedione

Fig. 2: Release of flavour compounds in the head space air from freshly brewed coffee. With the PTR-MS technology you can see aroma as you taste it! 
Clean Air Baseline Station on the volcanic Mauna Loa mountain. Today the Ionicon Team (see www.ptrms.com) manufacture and sell PTR-MS instruments throughout the world. Now more than 70 instruments are used by many research groups and companies around the world, applying this technique in various fields. Our customers include noted multinational companies and renowned research institutions in the fields of environmental science and food technology.

Today, three different PTR-MS instruments are available. Besides the standard and high sensitive versions, the compact PTR-MS is the most recent development from Ionicon Analytik. The compact PTR-MS, the first commercially available configuration for more general applications, is pictured in Figure 3.

\section{The future}

In our business it is crucial to always be innovative. We therefore want to promote collaborative research between universities and industry. Ionicon Analytik is a partner in ISONET a Marie Curie Research and Training Network recently funded by the European Commission (http://imk-ifu.fzk.de/isonet). One objective of ISONET is to integrate an industrial partner to strengthen collaboration between industry and academia and to allow network trainees to use state-of-the-art and innovative facilities to carry out their research.

The goal of the $2^{\text {nd }}$ International PTR-MS Conference next year (www.ptrms-conference.com) is to bring together active scientists and technologists involved in real-world mass spectrometric measurements of VOC from both academia and industry. The intent to found and organise such a biennial meeting was to promote discussion and stimulate the free exchange of ideas across disciplines, such as environmental sciences, food technology and medicine

\section{About the author}

Armin Hansel is Associate Professor in Physics at the University of Innsbruck. His research interests are ion-molecule-reactions and their application to trace gas analysis, which resulted in the development of the PTR-MS technique. In environmental physics he was involved in several international field programs. Armin is founding member of the academic spin-off company Ionicon Analytik GmbH.

\section{References}

[1] A. Hansel et al. (1995), Proton transfer reaction mass spectrometry: on-line trace gas analysis at ppb level, Int. J. of Mass Spectrom. and Ion Processes, 149/150, 609-619.

[2] W. Lindinger, A. Hansel, and A. Jordan, (1998) Proton-transferreaction mass spectrometry (PTR-MS): on-line monitoring of volatile organic compounds at pptv levels, Chemical Society Reviews, 27, 347-354.

[3] J. Lelieveld (2001), et al. The Indian Ocean Experiment: Widespread Air Pollution from South and Southeast Asia, Science, 291, 1031-1036.

[4] B.P. Wert, et al. (2003), Signatures of Alkene Oxidation in Airborne Formaldehyde Measurements DuringTexAQS 2000, J. Geophys. Res.108(D3), 4104, doi: 10.1029/2002JD002502

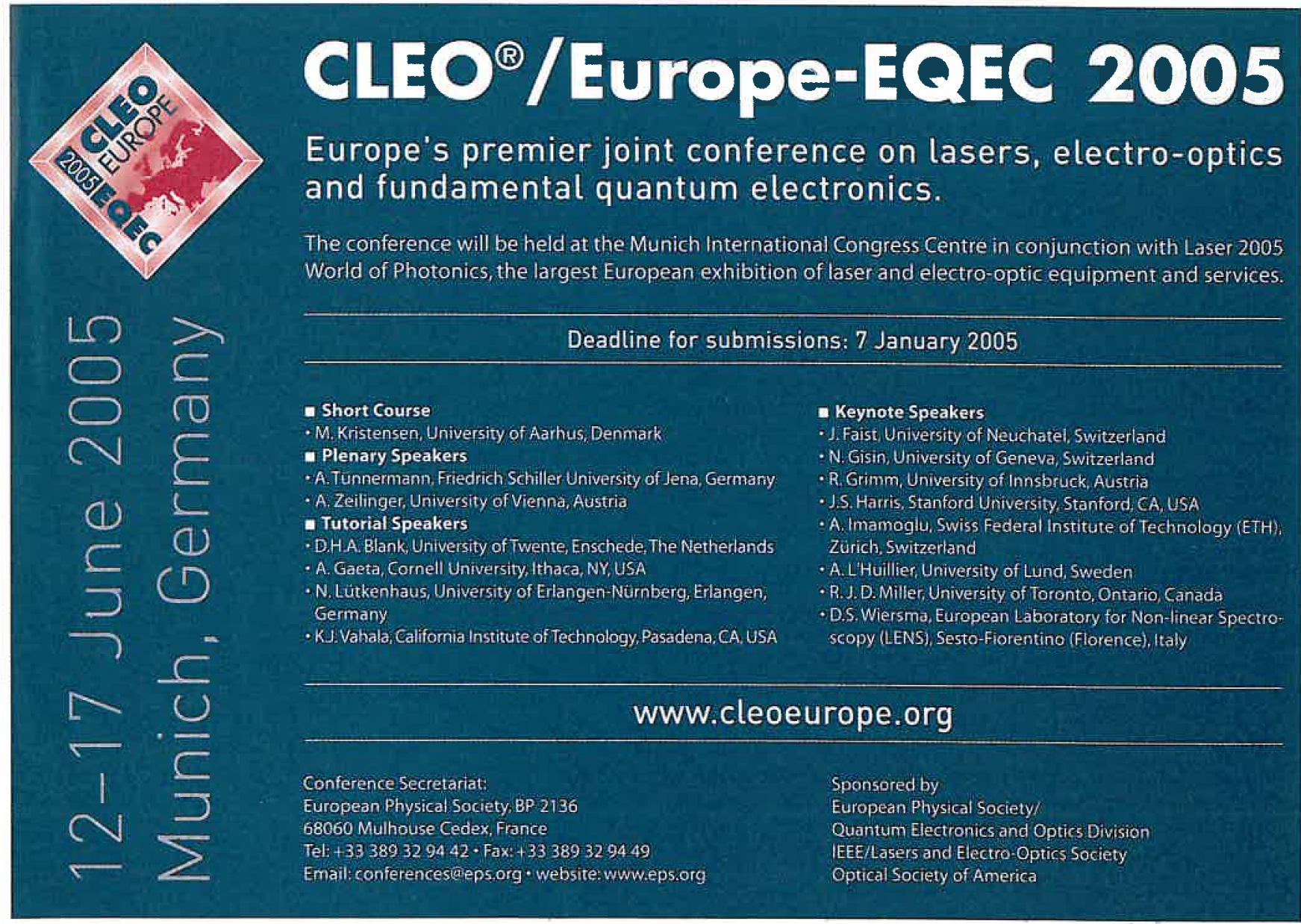

